\title{
Productividad primaria neta en una selva tropical húmeda
}

\author{
JAVIER ALVAREZ SÁNCHEZ ${ }^{1}$
}

RESUMEN. Estudio realizado en la selva alta perennifolia de Los Tuxtlas, Veracruz, se estimó la producción primaria neta (PPN) a través del muestreo de la caída de hojarasca en una hectárea de selva, llevado a cabo durante tres años. La PPN fue de 10.6, 13.2 y 16.2 $\mathrm{t} \mathrm{ha}^{-1}$ año ${ }^{-1}$, respectivamente. Estos valores están por debajo del promedio registrado para este tipo de comunidades, ya que aún no se ha medido la productividad de raíces en esta selva mexicana. Lo anterior se considera en relación a los recursos disponibles en el suelo, así como en función de la influencia de las características climáticas en la región.

\begin{abstract}
The net primary productivity (NPP) of a tropical rain forest in Los Tuxtlas, Veracruz, México, was determined sampling the litterfall in one ha of forest during a three year period. NPP was, for each of the three years 10.6, 13.2 and $16.2 \mathrm{t} \mathrm{ha}^{-1}$ year ${ }^{-1}$. These figures are lower than the mean values reported for these communities, however in the present study root productivity was not measured. Results are discussed in the light of soil resources availability and the influence of local climatic conditions.
\end{abstract}

El funcionamiento y estructura de un ecosistema está ligado estrechamente a la energía aprovechable y a su flujo a través de los componentes del mismo. La fuente principal de energía es el sol, el proceso de aprovechamiento la fotosíntesis y el flujo ocurre por el consumo, la descomposición y la evapotranspiración.

La producción primaria bruta (PPB) representa el efecto de la fotosíntesis en el ecosistema que se traduce parcialmente en la fijación de energía solar en materia orgánica nueva en un intervalo de tiempo dado. La cantidad de materia que se utiliza para la formación de tejidos y de substancias de reserva durante dicho período es la producción primaria neta (PPN) (Westlake, 1963; Jordan, 1983; Medina y Klinge, 1983).

\footnotetext{
${ }^{1}$ Departamento de Biología, Laboratorio de Ecología, Universidad Narional Autónoma de México, Delegación Coyoacán, 04510, México, D. F.
} 
Los componentes básicos de la productividad son el índice de área foliar, la tasa fotosintética neta, la proporción tallo-raíz y la longevidad de la hoja (Mooney y Gulmon, 1983). La variación de estos componentes, y por tanto de la productividad, está determinada por la variación de los recursos (nutrientes, agua y luz) disponibles en el sitio, resultando en este contexto muy importantes el efecto de los árboles del dosel sobre las tasas de extinción de la luz y el cambio en las condiciones microclimáticas. De lo anterior pueden resultar modificaciones importantes en la eficiencia fotosintética de las hojas, así como en la variación de las tasas respiratorias de los distintos tejidos vegetales (Jarvis y Leverenz, 1983).

Lo interesante en términos de disponibilidad, es que los recursos no son constantes y con ello puede variar la respuesta fotosintética de las plantas. En términos generales, las especies que colonizan áreas perturbadas tienen una tasa fotosintética alta, y la elevada disponibilidad de recursos permite que respondan con un crecimiento acelerado a través de un patrón de acumulación de nutrientes determinado fundamentalmente por la secuencia de sus eventos fenológicos, canalizando así más recursos a estructuras reproductivas (Mooney y Gulmon, 1983; Chapin, 1983); ésto no ocurre con plantas de estadios sucesionales tardíos, que dependen en mayor proporción de patrones estacionales de disponibilidad de recursos en el suelo.

La productividad primaria (la producción primaria por unidad de tiempo) depende de las condiciones particulares de un ecosistema, como son: cambios de temperatura, concentración de $\mathrm{CO}_{2}$, fertilidad del suelo, disponibilidad de agua y su estacionalidad, diferencias bioquímicas en el metabolismo, consumidores primarios y edad de las poblaciones (Etherington, 1975; Golley, 1979; Jordan, 1983; Jarvis y Leverenz, 1983).

La PPN se encuentra relacionada con el incremento de biomasa vegetal, puede obtenerse una estimación adecuada midiendo el aumento de peso en el tiempo. Parte de este material forma el tejido de estructuras que se pierden por senescencia fisiológica, como es el caso de las hojas. Por esta razón, en comunidades estables, se ha utilizado a la caída de hojarasca como un estimador de la PPN dado que no existe un incremento en la productividad neta del ecosistema (Medina y Klinge, 1983). Además, sería necesario considerar también las pérdidas por herbivoría, los árboles muertos en pie y la biomasa hipógea. En este trabajo se determinó de una manera indirecta la PPN a través del muestreo de la caída de hojarasca durante tres ciclos anuales en una hectárea de selva.

\section{ZONA DE ESTUDIO}

Se ubica dentro de los terrenos de la Estación de Biología Tropical Los Tuxtlas, Instituto de Biología, UNAM. La Estación se encuentra al sureste del estado de Veracruz, en una región de clima cálido húmedo con una precipitación promedio anual de $4700 \mathrm{~mm}$ y una temperatura media anual de $24.3^{\circ} \mathrm{C}$. 
Los suelos dominantes en el área son del tipo regosol eútrico. Lot-Helgueras (1976) y Estrada, et al. (1985) detallan información acerca de la altitud, geología y topografía de la zona.

La vegetación del lugar es una selva alta perennifolia (Miranda y Hernández X., 1963), para la cual se ha señalado que a nivel de una hectárea el único estrato que se puede delimitar está formado por palmas y arbustos (Bongers, et al., 1988). El 55\% de las especies (con altura $\geq 0.5 \mathrm{~m}$ ) son árboles, y solo una décima parte de todos los individuos con DAP $\geq 1.0 \mathrm{~cm}$ son deciduos. Se presenta un dosel cerrado entre los 30 y $35 \mathrm{~m}$ y estructuralmente se caracteriza por poseer una densidad de 2976 individuos con DAP $\geq 1.0 \mathrm{~cm}$ por hectárea. Entre las especies más importantes se encuentran: Astrocaryum mexicanum Liebm, la cual domina en el sotobosque, Pseudolmedia oxyphyllaria Donn. Sm., Nectandra ambigens (Blake) C.K. Allen, Dendropanax arboreus (L.) Dcne. \& Planch. y Poulsenia armata (Miq.) Standl.

La dinámica de regeneración de la selva se desarrolla a través de un complejo mosaico de distintas fases sucesionales. Este es creado principalmente por la caída de árboles y grandes fragmentos de ramas, sobre todo durante la época de vientos fuertes ("nortes"). De esta manera, existe una proporción de 0.4 de superficie ocupada por claros en 1.8 ha (Martínez-Ramos, 1985). La tasa de recambio de la comunidad (turnover rate) es de 97 años según Torquebiau (1981), mientras que Martínez-Ramos (1985) la calculó de 24 para el borde de la selva.

\section{Metodología}

Se usaron 100 trampas de nylon de $50 \mathrm{~cm}$ de diámetro para capturar la hojarasca, distribuídas sistemáticamente cada $10 \mathrm{~m}$ en una hectárea. El material se colectó mensualmente (en promedio) durante tres ciclos anuales en un período que abarcó de junio de 1981 a mayo de 1984, cada vez el material fue transportado al laboratorio donde fue separado después de secarlo a temperatura ambiente. Finalmente, se cuantificó el peso seco $\left(48\right.$ horas a $100{ }^{\circ} \mathrm{C}$ ) de las siguientes estructuras: hojas, flores, frutos, ramas y fragmentos no identificados (menores a $3 \mathrm{~mm}$ de longitud).

\section{RESULTADOS Y DISCUSIÓN}

La caída de la hojarasca alcanzó su valor más alto en la temporada seca. En promedio para los tres años, el $68 \%$ correspondió a hojas, $21.6 \%$ a ramas, $0.60 \%$ a flores, $3.1 \%$ a frutos y $6.8 \%$ a fragmentos no identificados (tabla 1 ).

La distribución temporal de la caída de estructuras reproductivas presentó un máximo en los meses de abril y mayo para las flores (hasta $0.15 \mathrm{~g} \mathrm{~m}^{-2} \mathrm{dia}^{-1}$ ), y de octubre a noviembre para los frutos (hasta $0.3 \mathrm{~g} \mathrm{~m}^{-2} \mathrm{dí}^{-1}$ ). Por lo que respecta a las ramas, los 
valores máximos ocurrieron durante los meses en que se presentan los "nortes" (hasta $1.9 \mathrm{~g} \mathrm{~m}^{-2} \mathrm{día}^{-1}$ ) (Alvarez Sánchez, 1988). El rango en cuanto a la caída de las estructuras foliares estuvo entre 4.9 y $0.5 \mathrm{~g} \mathrm{~m}^{-2} \mathrm{dí}^{-1}$, correspondiendo siempre el máximo al final de la sequía, cuando las especies están sujetas a una tensión hídrica severa, lo que provoca que el potencial hídrico en las hojas sea más negativo (Birk y Simpson, 1980; Leigh y Windsor, 1983); de hecho, Jordán (1983) ha señalado que la poca disponibilidad de agua provoca en los árboles una disminución en el crecimiento. Así, la caída de hojarasca en la temporada seca reduce la superficie foliar y la evapotranspiración, y por ello la diferencia de potenciales entre el suelo y la planta (Meentemeyer, et al., 1982).

En este sentido, otros estudios donde se ha determinado que la caída de hojarasca ocurre con mayor intensidad en la época seca son los de Bernhard (1970); Haines y Foster (1977); Kira (1978); Franken, et al. (1979); Kunkel-Westphal y Kunkel (1979); Wooi-Khoon y Jin-Eong (1983) y Spain (1984).

TABLA 1. Caída de las fracciones reconocidas de la hojarasca $\left(t h^{-1} \pm\right.$ desviación estándar) para cada ciclo anual en la selva de Los Tuxtlas, Veracruz.

\begin{tabular}{|c|c|c|c|c|}
\hline & $1981-1982$ & $1982-1983$ & $1983-1984$ & $\bar{x}$ \\
\hline Hojas & $\begin{array}{c}5.46 \pm 040 \\
(66.75)\end{array}$ & $\begin{array}{c}5.93 \pm 0.32 \\
(58.37)\end{array}$ & $\begin{array}{c}9.81 \pm 0.44 \\
(78.54)\end{array}$ & $\begin{array}{c}7.07 \\
(67.88)\end{array}$ \\
\hline Ramas & $\begin{array}{c}1.64 \pm 0.06 \\
(20.05)\end{array}$ & $\begin{array}{c}2.89 \pm 0.02 \\
(28.44)\end{array}$ & $\begin{array}{c}2.03 \pm 0.12 \\
(16.25)\end{array}$ & $\begin{array}{c}2.19 \\
(21.58)\end{array}$ \\
\hline $\begin{array}{l}\text { Fragmentos no } \\
\text { idenfificados }\end{array}$ & $\begin{array}{c}0.92 \pm 0.05 \\
(11.25)\end{array}$ & $\begin{array}{c}0.64 \pm 0.02 \\
(6.30)\end{array}$ & $\begin{array}{c}0.37 \pm 0.10 \\
(2.96)\end{array}$ & $\begin{array}{c}0.64 \\
(6.84)\end{array}$ \\
\hline Frutos & $\begin{array}{c}0.08 \pm 0.01 \\
(1.05)\end{array}$ & $\begin{array}{c}0.64 \pm 0.04 \\
(6.30)\end{array}$ & $\begin{array}{c}0.25 \pm 0.02 \\
(2.00)\end{array}$ & $\begin{array}{c}0.32 \\
(3.12)\end{array}$ \\
\hline Flores & $\begin{array}{c}0.08 \pm 0.02 \\
(0.98)\end{array}$ & $\begin{array}{c}0.06 \pm 0.01 \\
(0.59)\end{array}$ & $\begin{array}{c}0.03 \pm 0.003 \\
(0.24)\end{array}$ & $\begin{array}{c}0.06 \\
(0.60)\end{array}$ \\
\hline TOTAL & 8.18 & 10.16 & 12.49 & \\
\hline
\end{tabular}

El número entre paréntesis representa el porcentaje. 
Alvarez Sánchez (1988) señaló con base en los datos climatológicos en la selva de Los Tuxtlas que las temperaturas máximas extremas pueden presentarse desde el mes de marzo, prolongándose ocasionalmente hasta julio. Los coeficientes pluviométricos indican una deficiencia en la precipitación de diciembre a mayo, concentrándose el $50 \%$ de la precipitación total de enero hasta mediados del mes de julio. Es por ésto que el 43\% de la lluvia corresponde a un período húmedo de aproximadamente tres meses y medio mientras que en los tres meses de sequía sólo se registra el $7 \%$ de la precipitación total anual. Es en éste lapso donde se concentra casi el $50 \%$ de la caída total anual de hojarasca.

Bray y Gorham (1964) resumieron por primera vez los datos de producción de hojarasca en distintos ecosistemas en el mundo, con base a ellos Murphy en 1977 (citado en Jordan 1983) determinó a la PPN como el triple de aquella; en realidad, Jordan (1983) demostró que para las selvas tropicales la PPN es 1.3 veces la caída de hojarasca. Este último factor fue considerado para efectos de la estimación de PPN en este trabajo. En la tabla 2 se presenta PPN para algunas de las selvas tropicales, datos que fueron obtenidos utilizando este factor de 1.3. No obstante, debe considerarse que la colecta de hojarasca utilizando trampas subestima la producción real debido a la descomposición y a la pérdida de materia orgánica del sistema.

Para la selva mexicana a la que se hace mención en este estudio, la PPN fue de 10.6, 13.2 y $16.2 \mathrm{tha}^{-1}$ año $^{-1}$ para los tres ciclos de estudio, respectivamente. Estos valores se encuentran por debajo de las 28 toneladas promedio mencionadas por Lieth (1975) para las selvas tropicales, valor que incluye la producción de raíces, aspecto poco conocido y cuantificado en este tipo de comunidades. En el Amazonas, por ejemplo, fue de 1.17 $\mathrm{t} \mathrm{ha}^{-1}$ año $^{-1}$ (Jordán y Escalante, 1980), mientras que para la selva de los Tuxtlas la productividad de raíces se estimó en $0.2 \mathrm{t} \mathrm{ha}^{-1}$ año $^{-1}$ (Sánchez-Gallén y Alvarez Sánchez, datos no publicados).

Comparando la productividad aérea promedio anual de la selva de Los Tuxtlas con las demás (tabla 2), resultó superior en todos los casos a excepción de las selvas de Colombia (Foelster y De Las Salas, 1976), Panamá (Haines y Foster, 1977 y Golley, et al., 1975) y una de las localidades del estudio de Bernhard (1970) en Costa de Marfil. Sin embargo, Medina y Klinge (1983) señalan que la PPN supera las 20 toneladas en las selvas de Africa y Asia.

Según Jordan (1983), la productividad no varía apreciablemente entre selvas eutróficas (como quizá sería la de Los Tuxtlas debido a su origen volcánico, Estrada, et al., 1985) y oligotróficas (como la del Amazonas, Jordan y Herrera, 1981). En este sentido, Cuevas y Medina (1986) registran $10.25 \mathrm{t} \mathrm{ha}^{-1}$ año $^{-1}$ de caída de hojarasca en una localidad venezolana del Amazonas, mientras que en Manaus y Belem (Brasil) se obtuvo un total de caída de hojarasca de 6.7 y $9.0 \mathrm{t} \mathrm{ha}^{-1}$ año $^{-1}$, respectivamente Klinge en 1974 (citado en Jordan, 1983).

Jordan y Herrera (1981), mencionan que los mecanismos de conservación de nutrientes son la razón por la cual los valores de productividad no difieren mucho entre 
TABLA 2. Valores de productividad primaria neta (PPN) determinados a partir de la caída de hojarasca en selvas tropicales húmedas del mundo.

\begin{tabular}{|c|c|c|c|c|}
\hline País & $\left(\mathrm{t} \mathrm{ha}^{-1}\right.$ año $\left.^{-1}\right)$ & $\begin{array}{l}\text { Altitud } \\
\text { (msnm) }\end{array}$ & $\begin{array}{l}\text { Precipitación } \\
\qquad(\mathrm{mm})\end{array}$ & Bibliografía \\
\hline México & $\begin{array}{l}10.6 \\
13.2 \\
16.2\end{array}$ & 150 & 4707 & Este trabajo \\
\hline Nueva Guinea & 9.8 & 2450 & 3985 & Edwards (1977) \\
\hline Guatemala & $\begin{array}{l}12.1 \\
13.1\end{array}$ & $\begin{array}{r}900 \\
1000\end{array}$ & $\begin{array}{l}3747 \\
3747\end{array}$ & $\begin{array}{l}\text { Kunkel-Westphal } \\
\text { y Kunkel (1979) }\end{array}$ \\
\hline Australia & 12.6 & 60 & 3609 & Spain (1984) \\
\hline Venezuela & 12.4 & 50 & 3521 & $\begin{array}{l}\text { Jordan y Esca- } \\
\text { lante (1980) }\end{array}$ \\
\hline Guyana & 11.2 & 50 & 3373 & Puig (1979) \\
\hline Trinidad & $\begin{array}{l}8.9^{*} \\
9.1\end{array}$ & $\begin{array}{r}70 \\
200\end{array}$ & $\begin{array}{l}2900 \\
3300\end{array}$ & Cornforth (1970) \\
\hline Jamaica & $\begin{array}{l}6.2^{* * *} \\
7.4\end{array}$ & 510 & $\begin{array}{l}3080 \\
2600\end{array}$ & $\begin{array}{l}\text { Wiegert (1970) } \\
\text { citado en Spain (1984) }\end{array}$ \\
\hline Colombia & $\begin{array}{l}15.6 \\
11.3\end{array}$ & $\begin{array}{l}75 \\
75\end{array}$ & $\begin{array}{l}3000 \\
3000\end{array}$ & $\begin{array}{l}\text { Foelster y de } \\
\text { Las Salas (1976) }\end{array}$ \\
\hline Panamá & 14.4 & 137 & 2725 & $\begin{array}{l}\text { Haines y Foster } \\
\text { (1977) }\end{array}$ \\
\hline Puerto Rico & 8.6 & 1586 & 2600 & Tanner (1980) \\
\hline Australia & 12.8 & 700 & 2544 & $\begin{array}{l}\text { Brassel, et al. } \\
\text { (1980) }\end{array}$ \\
\hline Panamá & 13.6 & 425 & 2500 & Golley, et al (1975) \\
\hline Brasil & $\begin{array}{l}12.9^{* *} \\
8.3\end{array}$ & $\begin{array}{l}10 \\
45\end{array}$ & $\begin{array}{l}2277 \\
1771\end{array}$ & $\begin{array}{l}\text { Franken, et al. } \\
\text { (1979) }\end{array}$ \\
\hline Malasia & 12.0 & 112 & 2054 & $\begin{array}{l}\text { Ogawa (1978) y } \\
\text { Lim (1978) citado en } \\
\text { Spain (1984) }\end{array}$ \\
\hline Guatemala & 11.7 & 100 & 2000 & Ewel (1976) \\
\hline Costa de Marfil & $\begin{array}{l}12.4^{* *} \\
12.1\end{array}$ & $\begin{array}{r}100 \\
50\end{array}$ & $\begin{array}{l}1800 \\
2100\end{array}$ & Bernhard (1970) \\
\hline Ghana & 12.6 & 152 & 1650 & John (1973) \\
\hline Brasil & $6.0^{*}$ & 850 & 1566 & Jackson (1978) \\
\hline Zaire & 11.9 & 1208 & 1273 & $\begin{array}{l}\text { Malaisse, et al. } \\
\text { citado en }\end{array}$ \\
\hline & 7.7 & 1190 & 1279 & Spain (1984) \\
\hline Nigeria & 6.0 & 135 & 1321 & Hopkins (1966) \\
\hline Nigeria. & 7.3 & 250 & 1200 & Madge (1965) \\
\hline
\end{tabular}

* Considerando únicamente la fracción foliar.

** Distintas localidades de estudio. 
estas selvas. Estos mecanismos son: el desarrollo exhuberante de una red de raíces finas secundarias, la captación de nutrientes a través de micorrizas, el desarrollo de hojas esclerófilas y su arreglo geométrico en el suelo, a los que puede añadirse el crecimiento de epífitas, algunas de las cuales pueden (por medio de microorganismos asociados) fijar nitrógeno directamente de la atmósfera, así como el rápido establecimiento y desarrollo de la vegetación secundaria (Herrera, et al., 1978; Herrera, et al., 1978a). Según Jordan y Herrera (1981) no debería esperarse que estos mecanismos fueran tan importantes en ecosistemas eutróficos.

Gentry (1983) propone que otro factor importante que se ha olvidado y subestimado en el análisis de la productividad tropical, es la contribución de las lianas. En éstas se ha eficientizado el crecimiento vegetativo ya que alcanzan el dosel invirtiendo poca energía en el tronco que las sostiene, por lo que su relación biomasa foliar/leñosa es mayor. El autor estima que las lianas contribuyen con un tercio del total de la hojarasca en las selvas tropicales. En la selva de Los Tuxtlas, tomando en cuenta que seguramente existen varias especies de lianas entre los morfos que no han sido identificados, las lianas y bejucos más importantes promediaron $3.7 \%$ con respecto al total de la caída de hojas (Alvarez Sánchez 1988), datos que se alejan rıucho de la propuesta de Gentry.

La productividad está limitada desde luego por la energía solar y en buena proporción por los recursos disponibles en ei sitio, principalmente agua y nutrientes. De esta manera, tanto la capacidad fotosintética como la duración de la vida de la hoja están limitados por factores ambientales (Mooney y Gulmon, 1983). Dado que regiones con climas similares podrían considerarse como áreas con recursos semejantes aunque la respuesta de las especies a éstos últimos fuera distinta, es posible hipotetizar que existe una convergencia en la productividd en zonas apartadas considerando que los componentes fisiológicos de la misma responden a la variación en dichos recursos (Mooney y Gulmon, 1983).

Resulta difícil encontrar parámetros que permitan establecer la validez de estas comparaciones, ya que las especies responden de manera diferente a las condiciones microambientales y existen variaciones en altitud entre los sitios. Además, la estimación de la PPN a través de la caída de hojarasca depende fuertemente del tiempo de observación debido a la influencia que tiene la caída de distintas cohortes de hojas de vida larga. Por otra parte, si las metodologías han sido distintas, debe tenerse mucho cuidado en la comparación de los valores de productividad entre distintos ecosistemas (Proctor, 1983).

AGRADECIMIENTOS. Se agradece al Dr. Sergio Guevara del Laboratorio de Ecología, Facultad de Ciencias, UNAM, los valiosos comentarios que hizo al trabajo. 


\section{LITERATURA CITADA}

AlVAREZ SÁNCHEZ, F. J. 1988. Estimación de la caída y descomposición de la hojarasca y su relación con la dinámica de una selva mexicana. Tesis Doctoral, Facultad de Ciencias, UNAM, México, D.F. $105 \mathrm{p}$.

BERNHARD, F. 1970. Etude de la litière et de sa contribution au cycle des èlèments minéraux en forét ombrophile de Côte-D'Ivoire. Oecol. P1. 5:247-266.

BIRK, E. y R. SIMPSON. 1980. Steady state and the continuous imput model of litter accumulation and decomposition in Australian eucalypt forest. Ecology 61 (3):481-485.

Bongers, F., J. PopMA, J. MEAVE y J. CARABIAS. 1988. Structure and floristic composition of the lowland rain forest of Los Tuxtlas, Mexico, Vegetatio 74:55-80.

BRASSELL, H., G. UNWIN y G. STOCKER. 1980. The quantity temporal distribution and mineral element content of litter fall in two forest types at two sites in tropical Australia. J. Ecol. 68:123-139.

BRAY, J. y E. GORHAM. 1964. Litter production in forests of the world. Adv. Ecol. Res. 2:101-157.

CONFORTH, J. 1970. Leaf fall in a tropical rain forest J. Appl. Ecol. 7:603-608.

CUEVAS, E. y E. MEDINA. 1986. Nutrient dynamics within amazonian forest ecosystems. I. Nutrient flux in fine litter fall and efficiency of nutrient utilization. Oecologia 68(3):466-472.

CHAPIN, F. 1983. Patterns of nutrient absorption and use by plants from natural and man-modified environments. En: Mooney, H. y M. Godraw (Edrs.). Disturbance and ecosistems components of response. Springer-Verlag, Berlin. Ecological Studies 44 pp. 175-187.

EDWARDS. P.J. 1977. Studies of mineral cycling in a montage rain forest in New Guinea. II. The production and disappearance of litter. J. Ecol. 65:971-992.

EstradA, A., R, CoATES-ESTRADA y M. MARTÍNEZ-RAMOS. 1985. La Estación de Biología Tropical Los Tuxtlas: un recurso para el estudio y conservación de las selvas del trópico húmedo. En: Gómez-Pompa, A. y S. del Amo (Edrs.). Investigaciones sobre la regeneración de selvas altas en Veracruz, México. Alhambra, México. v. II. pp. 379-393.

ETHERINGTON, J. 1975. Environment and plant ecology, John Wiley \& Sons., Great Britain.

EWEL, J. J. 1976. Litter fall and leaf decomposition in a tropical forest succesion in eastern Guatemala. J. Ecol. 64:293-308.

FOELSTER, A. y G. DE LAS SALAS. 1976. Litter fall and mineralization in three tropical evergreen forest stands, Columbia. Acta. Ci. Venez. 21:196-202.

FranKEN, M., V. IRMLER y H. KLINGE. 1979. Litter fall in inundation riverine and "Terra Firme" forest of Central Amazonia. Trop. Ecol. 20(2):225-235.

GENTRY, A. 1983. Lianas and the "paradox" of the contrasting latitudinal gradients in the wood and litter production. Trop. Ecol. 24(1):63-67.

Golley, F., J. MC. Ginnis, R. Clements, G. Child y M. Deuver. 1975. Mineral cycling in a tropical moist forests ecosystems. University of Georgia Press.

GOLLEY, F. 1979. Production primaire brute et nette, parametres de croissance. Dans: UNESCO. Recherches sur les ressources naturelles: ecosistemes forestiers tropicaux. UNESCO, PNE, FAO, France. pp. 252-268.

HAINES, B. y R. FOSTER. 1977. Energy flow through litter in a Panamanian forest. J. Ecol. 65:147-155.

HERRERA, R., C. JoRDAN, H. KLINGE y E. MEDINA. 1978. Amazon ecosystems, their structure and function with particular emphasis on nutrients. Interciencia 3(4):223-232.

HerRerA, R., T. MERIDA, N. STARK y C. JORDAN. 1978a. Direct phosporus transfer from leaf litter to roots. Naturwissenschaften 65:208-209.

HOPKINS, B. 1966. Vegetation in Olokemeji Forest Reserve, Nigeria. IV. The litter and soil with special reference to their seasonal changes. J. Ecol. 54:647-703.

JACKSON, J. 1978. Seasonality of flowering and leaf fall in a Brazilian subtropical lower montane mois: forest. Biotropica 10(1):38-42. 
JARVIS, P. y J. LEVERENZ. 1983. Productivity in temperate deciduos and evergreen forest. En: Lange, O.L. et al.(Edrs.). Encyclopedia of plant physiology. Springer-Verlag, Berlin. New Series v. 12.

JOHN, T. 1973. Accumulation and decay of litter and net production of forest in tropical West Africa. Oikos 24:430-435.

JORDAN, C. y G. ESCALANTE. 1980. Root productivity in an Amazonian rain forest. Ecology 61(1):14-28.

JORDAN, C., y R. HERRERA. 1981. Tropical rain forest: are nutrients really critical?. Amer. Nat. 117(2):167-180.

JORDAN, C. 1983. Productivity of tropical rain forest ecosystems and the implications for their use as future wood and energy sources. En: Golley, F. (Edr.) Tropical rain forest ecosystems. Elsevier, Amsterdam. pp. 117-136.

JORDAN, C. 1983a. Nutrient regime in the wet tropics: physical factors. En: Medina, E. et al. (Edr.) Physiologycal ecology of plants in the wet tropics. Dr. W. Junk Publ., The Hague.

KIRA, T. 1978. Community architecture and organic matter dynamics in tropical lowland rain forest of southeast Asia with special reference to Pasoh Forest, West Malaysia. En: Tomlinson P. y M. Zimmermann (Edrs.). Tropical trees as living systems. Cambridge Univesity Press, New York. pp. 561-590.

KUNKEL-WESTPHAL, I. y P. KUNKEL. 1979. Litter fall in a Guatemalan primary forest, with details of leaf-shedding by some common tree species. J. Ecol. 67:665-686.

LEIGH, E. y D. WINDSOR. 1983. Forest production and regulation of primary consumers on Barro Colorado Island. En: Leigh, E.S. y D. Windsor (Edrs.). The ecology of a tropical forest. Seasonal rhythms and long-term changes. Smithsonian Institution Press, Washington, D.C. pp. 355-387.

LIETH, H. 1975. Primary productivity in ecosystems: comparative analysis of global patterns. Unifing concepts in Ecology. Rept. Plenary sesions. First. Internat. Cong. Ecol. The Hague, Netherlands 1974. W. H. Van Dobben y R.H. Lowe-McConnell (Edrs.) The Hague: W. Junk B.V. and Wageningen. Centre for Agricultural Publishing and Documentation. pp. 67-88.

Lot-Helgueras, A. 1976. La Estación de Biología Tropical Los Tuxtlas: pasado, presente y futuro. En: Gómez-Pompa, A., et al. (Edrs.). Investigaciones sobre la regeneración de selvas altas en Veracruz, México. INIREB, CECSA, México, D.F. v.1. pp. 31-69.

MADGE, D. 1965. Litterfall and litter disappearance in a tropical forest. Pedobiologia 55:273-278.

MARTÍNEZ-RAMOS, M. 1985. Claros, ciclos vitales de los árboles tropicales y regeneración natural de las selvas altas perennifolias. En: Gómez-Pompa, A. y S. del Amo (Edrs.). Investigaciones sobre la regeneración de las selvas altas en Veracruz, México. INIREB, Alhambra, México, D.F. v.II. pp. 191-239.

MEdinA, E. y H. KLINGE. 1983. Productivity of tropical forests and tropical woodlands. En: Lange, D.L. et al. (Edrs.). Encyclopedia of plant physiology. Springer-Verlag, Berlin. New Series. v. 12D.

MEENTEMEYER, V., E. BOX y R. THOMPSON. 1982. World patterns and amounts of terrestrial plant litter production. Bioscience 32(2): 125-128.

Miranda, F. y E. HERnANDEZ X. 1963. Los tipos de vegetación de México y su clasificación. Bol. Soc. Bot. México 28:29-72.

MOONEY, H. y S. GULMON. 1983. The determinants of plant productivity natural versus man-modified communities. En: Mooney, H. y M. Boddraw (Edrs.). Disturbance and ecosystems components of response. Springer-Verlag, Berlin. Ecological Studies 44. pp.146-158.

PROCTOR, J. 1983. Tropical forest litter fall. I. Problems of data comparison. En: Sutton, S.C., et al. (Edr.). Tropical rain forest: ecology and management. Blackwell Scientific Publications, Leeds, pp. 267-273.

PuIG, H. 1979. Production de litiêre en forêt Guyanaise: resultats preliminaiaires. Ext. Bull. Soc. D'Hist. Nat. Toulouse $115(3-4)$.

SPAIN, A.V. 1984. Litterfall and the standing crop of litter in three tropical Australian rain forests. J. Ecol. 72:947-961. 
TANNER, E. 1980. Litter fall in montane rain forest of Jamaica and its relation to climate. J. Ecol. 68:833-848.

TORQUEBIAU, E. 1981. Analyse architectural de la fôret de Los Tuxtlas (Veracruz), Mexique. Tesis (Doctorado del Tercer Ciclo). Université des Sciences et Techniques du Languedoc, Montpellier.

WESTLAKE, D. 1963. Comparisons of plant productivity. Biol. Rev. 38:385-425.

WOOI-KHOON, G. y O. JIN EONG. 1983. Litter production and decomposition in a coasted hill dipterocarp forest. En: Sutton, S.C. et al. (Edrs.). Tropical rain forest: ecology and management Blackwell Scientific Publications, Leeds. pp. 275-285. 